\title{
When to Consider an Inborn Error of Metabolism in Adults?
}

\author{
Martijn C.G.J. Brouwers ${ }^{\mathrm{a}, \mathrm{b}, \mathrm{c}}$
}

\begin{abstract}
Inborn errors of metabolism (IEM) are a group of disorders that are often erroneously assumed to be the playing field of pediatricians only. This misconception combined with the rare nature of IEMs explains why there is often a serious delay in the diagnosis of an adult-onset IEM, which can have detrimental consequences. This review provides potential strategies to overcome this diagnostic delay. The most promising consists of stimulating critical clinical reasoning and creating awareness of the process that leads to the diagnosis of a rare disease.
\end{abstract}

Keywords: Inborn error of metabolism; Adult-onset; Clinical reasoning; Diagnosis; Metabolic disease

\section{Introduction}

Inborn errors of metabolism (IEM) are a heterogeneous group of 1,450 disorders that are individually rare to ultra-rare [1], but relatively common as a group (estimated birth prevalence $1: 2,000[2]$ ). Although genetic by definition, it is a misconception that IEMs only manifest at young age. Sometimes it takes years before a toxic metabolite accumulates (e.g., lysosomal storage disorder, Wilson's disease) [3], or an individual is predisposed to extreme environmental stressors (e.g., marathon, pregnancy) leading to metabolic decompensation (e.g., hyperammonemia, rhabdomyolysis) $[4,5]$. It has been estimated that $45 \%$ of the patients at the adult metabolic centers have been diagnosed at adult age [6]. Because of its rare character there is often a delay in the diagnosis of an IEM, which may sometimes lead to irreversible damage or even death. It is, therefore, imperative that a diagnosis is made more rapidly.

In the present paper, which will be introduced by a clinical

Manuscript submitted May 11, 2021, accepted June 21, 2021

Published online July 25, 2021

${ }^{a}$ Division of Endocrinology and Metabolic Disease, Department of Internal Medicine, Maastricht University Medical Centre, Maastricht, the Netherlands ${ }^{b}$ CARIM School for Cardiovascular Diseases, Maastricht University, Maastricht, the Netherlands

${ }^{\mathrm{c} C}$ Corresponding Author: Division of Endocrinology and Metabolic Disease Department of Internal Medicine, Maastricht University Medical Centre, P Debyelaan 25, 6229 HX Maastricht, the Netherlands.

Email: Mcgj.brouwers@mumc.nl

doi: https://doi.org/10.14740/jem750 case, potential approaches to reduce the diagnostic delay will be reviewed and tools will be offered.

\section{Clinical Case}

A 40-year-old woman was referred to the neurologist. She initially presented at the psychiatry department with confusion, apraxia and a slurred speech during a period of emotional exhaustion. The psychiatrist already performed standard laboratory workup, which did not reveal any abnormalities. Upon neurological investigation, the patient indeed exhibited mild signs of apraxia and a slurred speech. An additional electroencephalography (EEG) revealed slow wave activities, but no further abnormal signs. The patient was diagnosed with atypical psychosis and referred back to the psychiatrist.

One year later, the patient experienced a very similar episode, and again EEG showed slow wave activities. The neurologist now decided to check serum ammonia levels, which were strongly elevated $(160 \mu \mathrm{mol} / \mathrm{L})$. After liver failure was excluded, an IEM was suspected. Serum glutamine and urinary orotic acid levels were indeed elevated and patient was eventually diagnosed with ornithine transcarbamylase deficiency, an urea cycle disorder. She was treated with a protein-restricted diet, citrulline supplementation and a nitrogen scavenger (phenylbutyrate), which prevented further deterioration as well as new episodes of metabolic decompensation.

\section{Epicrisis: Premature Closure}

This is an illustrative case of an adult-onset IEM, which was diagnosed 1 year after first presentation. Unlike several previous case reports on adult-onset urea cycle defects [7], clinical outcome was fortunately good. To better understand what may have caused this diagnostic delay, it is important to provide some background on the process of clinical reasoning.

There are several models of clinical reasoning. The currently prevailing theory, the dual-process model, assumes there are two parallel modes of thinking, i.e., intuitive, heuristic (type 1) and reflective, analytic (type 2), as reviewed in [8]. Type 1 is fast, automatic and unconscious, whereas type 2 is slow and conscious, and requires more effort. Type 1 thinking is particularly prone to cognitive bias, which can lead to diagnostic errors [8]. Premature closure, i.e., the failure to consider reasonable alternatives after an initial diagnosis has been made, is the most frequently encountered type of cognitive bias [9], as was also the case in the current example. Although 
Table 1. Bayes Theorem Illustrated in an Example of Mitochondrial Disease

\begin{tabular}{|c|c|c|c|}
\hline & Mitochondrial disease present & Mitochondrial disease absent & \\
\hline \multicolumn{4}{|c|}{ Example $1^{\mathrm{a}}$ : low a priori chance } \\
\hline Lactate positive & 8 & 691 & PPV: 0.01 \\
\hline Lactate negative & Sensitivity: 0.77 & Specificity: 0.93 & Prevalence: 0.001 \\
\hline \multicolumn{4}{|c|}{ Example $2^{\text {b}}$ : high a priori chance } \\
\hline Lactate negative & Sensitivity: 0.77 & Specificity: 0.93 & Prevalence: 0.05 \\
\hline
\end{tabular}

${ }^{a}$ Example 1: Screening for a mitochondrial disease by using serum lactate levels (sensitivity $77 \%$, specificity $93 \%$ [14]) in a patient population presenting with fatigue (estimated prevalence of mitochondrial disease, assuming that all patients with mitochondrial disease are fatigued: 1:1,000 [15, 16]) yields an unacceptably low positive predictive value (PPV); the chance of having a mitochondrial disease with elevated serum lactate is still only 1:100. bxample 2: The use of serum lactate as a diagnostic test for mitochondrial disease in carefully selected patients (i.e., the presence of diabetes and hearing impairment [17]) yields a much better PPV; the chance of having a mitochondrial disease with elevated serum lactate is 1:3, modified after [18]. PPV: positive predictive value; NPV: negative predictive value.

slow wave activities on an EEG can be indicative of a toxic or metabolic encephalopathy [10], the neurologist initially ignored this finding and the patient was diagnosed with atypical psychosis. Apparently, an atypical presentation of a common disorder "won" from a typical presentation of a rare disorder. So how can this conundrum be solved?

\section{Should We Educate? The Forgetting Curve}

One immediate reflex could be that we should invest in teaching about these IEMs. This solution, however, has two drawbacks. First, as Ebbinghaus already demonstrated in 1885, almost $75 \%$ of what is taught will be been forgotten after $48 \mathrm{~h}$ [11]. Second, and even more problematic, as already stated in the introduction, 1,450 inborn errors of metabolism have been described. So, if there would be any memory retention on one or a handful of IEMs, it would be at the expense of all the other IEMs that equally deserve our attention.

One exception should be those treatable IEMs that can manifest as an acute, life-threatening metabolic decompensation, such as a post-partum hyperammonemia in a previously undiagnosed urea cycle defect [7].

\section{Should We Use Diagnosis Support Systems? Will It Be Used?}

An alternative solution to reduce the delay by which an IEM is diagnosed, is the use of a diagnosis support system. Currently, several support systems for the diagnosis of rare diseases have been developed, using different methodologies including artificial intelligence. The Inborn Errors of Metabolism Knowledgebase (IEMbase) is an expert-generated, web-based database that has specifically been designed for IEMs [12]. The algorithm allows input of both biochemical and clinical features and performs fairly well. Eighty-six percent of the cases is ranked within the top five of most likely diagnoses [12]. Notwithstand- ing the potential benefit of this diagnosis support system, it can be questioned whether this tool will solve the issue that was raised in the clinical case description. As long as the neurologist is confident of his initial diagnosis and not aware of an alternative, rare diagnosis, a diagnosis support system will not be consulted. This misalignment between the confidence in the diagnosis and the correctness of this diagnosis is not uncommon (25-41\%) and independent of clinical experience [13].

\section{Should We Screen? Bayes' Theorem}

A complete different approach, if we accept that general clinicians are not able to acquire all the knowledge on IEMs, and diagnostic support systems will not be consulted, could be that we should perform standard screening for IEMs in specific patient populations. For instance, we could measure serum ammonia levels in all patients with a psychosis to facilitate early diagnosis of urea cycle defects. However, the low a priori chance of an IEM in such populations will yield low and often unacceptable positive predictive values (PPV), even despite good diagnostic test characteristics. This often counterintuitive phenomenon, an example of Bayes' theorem, is further illustrated in an example of mitochondrial disease, Table 1 [14-18]. Of note, low PPVs and consequently high false-positive rates may be accepted if the clinical consequences of missing a diagnosis are high, as is the case with urea cycle defects.

The introduction of whole exome (and genome) screening has prompted researchers to evaluate this novel tool for screening in newborns, the ultimate way to yield an early diagnosis. A recent study, however, has shown that this approach currently does not perform better than conventional metabolite screening by tandem mass spectrometry in dried blood spots [19]. Furthermore, Bayes' theorem can also be applied to genetic screening: the a priori chance that a variant of unknown significance is truly pathogenic is much lower when all newborns are screened, in comparison to genetic testing in selected cases. Finally, another potential drawback of genetic screen- 
Table 2. General Principles That Should Be Taught in Order to Facilitate Early Diagnosis of an Inborn Error of Metabolism in Adults

Diagnosing an IEM is not restricted to pediatricians, some IEMs typically manifest at adult age.

Given the a low a priori chance of an IEM it is not advocated to screen for these disorders in the general population, as it will yield unacceptably high false positive rates. Instead, diagnostic tests should be reserved for selected cases.

An IEM (or another rare disease) should be considered when there is an atypical presentation of a common clinical problem ("question mark"); an IEM should be suspected when there are additional guiding signs ("plus sign"). Identification of question marks and plus signs can be stimulated by reflective reasoning.

When an IEM is suspected, the following steps can be undertaken to make the final diagnosis: 1) use (online) reference works; 2) use diagnosis support system; 3) initiate a multidisciplinary meeting; or 4) consult an expert. In this stage, exclusion of potential diagnoses because of low likelihood should be avoided.

ing, similar to metabolite screening, is that the penetrance, i.e., the clinical expression, of a pathogenic mutation is often not $100 \%$. A genetic diagnosis does not necessarily imply a clinical diagnosis. Newborn genetic screening, may therefore, not only prevent irreversible organ damage, but also result in uncertain future perspectives in young patients and their parents.

\section{Clinical Reasoning: Question Marks, Plus Signs and Reflective Reasoning}

The true solution for avoiding delays in diagnosing an IEM may lie in stimulating critical clinical reasoning and creating more awareness of the diagnostic process. Critical clinical reasoning implicates high alertness when there is an atypical presentation of a common clinical problem, a so-called question mark. This is the moment that an IEM should be considered. It does not require any background knowledge on IEMs, but instead, experience on typical presentations of common disorders. The presence of a question mark should prompt the clinician to search for additional signs that could guide towards a specific diagnosis, a so-called plus sign. When these principles are applied to the hitherto presented clinical case, there was a clear question mark that prompted the psychiatrist to refer the patient to the neurologist, i.e., the presence of apraxia and a slurred speech in a confused patient, in other words, an atypical presentation of a relatively common clinical problem. The abnormal EEG is an example of a plus sign.

The likelihood of correctly identifying question marks and plus signs, and thereby preventing premature closure and promoting consideration of an alternative diagnosis, can be increased by reflective reasoning, which consists of the following steps: 1) go back to the case and the initial diagnosis; 2) list the findings in favor of the initial diagnosis; 3) list the findings that are against the initial diagnosis; 4) list the findings that would be expected if the initial diagnosis would be true, but are not present [20]. Reflective reasoning, which is a method that stimulates type 2 , analytic clinical reasoning, has been shown to be effective, in particular in complex cases [21].

The co-existence of a question mark and plus sign should raise high suspicion of an IEM, and hence, justifies proceeding to the next step in the diagnostic process, which could consist of: 1) consulting (online) reference works; 2) using a diagnosis support system; 3) initiating a multidisciplinary meeting; and 4) consulting an expert. The pitfall that a potential diagnosis can be refuted because of a low likelihood should be avoided, since the differential diagnosis at this stage consists of only rare or atypical diagnoses. Instead, the complete list of potential diagnoses should be scrutinized.

\section{Conclusions}

A delay in the diagnosis of an IEM can have detrimental consequences. Currently, there is no magic bullet that can overcome this diagnostic conundrum. One solution may be that we teach about general principles that are necessary to diagnose an IEM and other rare disorders (Table 2), which should stimulate critical clinical reasoning and create awareness of the diagnostic process. These general principles should be reiterated at all stages of clinical training and are preferable to teaching about all individual IEMs.

\section{Acknowledgments}

None to declare.

\section{Financial Disclosure}

None to declare.

\section{Conflict of Interest}

None to declare.

\section{Data Availability}

The author declares that data supporting the findings of this study are available within the article.

\section{Abbreviations}

EEG: electroencephalography; IEM: inborn errors of metabolism; PPV: positive predictive value 


\section{References}

1. Ferreira CR, Rahman S, Keller M, Zschocke J, ICIMD Advisory Group. An international classification of inherited metabolic disorders (ICIMD). J Inherit Metab Dis. 2021;44(1):164-177.

2. Waters D, Adeloye D, Woolham D, Wastnedge E, Patel S, Rudan I. Global birth prevalence and mortality from inborn errors of metabolism: a systematic analysis of the evidence. J Glob Health. 2018;8(2):021102.

3. Meikle PJ, Hopwood JJ, Clague AE, Carey WF. Prevalence of lysosomal storage disorders. JAMA. 1999;281(3):249254.

4. Ben-Ari Z, Dalal A, Morry A, Pitlik S, Zinger P, Cohen J, Fattal I, et al. Adult-onset ornithine transcarbamylase (OTC) deficiency unmasked by the Atkins' diet. J Hepatol. 2010;52(2):292-295.

5. Hoffman JD, Steiner RD, Paradise L, Harding CO, Ding L, Strauss AW, Kaplan P. Rhabdomyolysis in the military: recognizing late-onset very long-chain acyl Co-A dehydrogenase deficiency. Mil Med. 2006;171(7):657-658.

6. Sirrs S, Hollak C, Merkel M, Sechi A, Glamuzina E, Janssen MC, Lachmann R, et al. The frequencies of different inborn errors of metabolism in adult metabolic centres: report from the SSIEM adult metabolic physicians group. JIMD Rep. 2016;27:85-91.

7. Arn PH, Hauser ER, Thomas GH, Herman G, Hess D, Brusilow SW. Hyperammonemia in women with a mutation at the ornithine carbamoyltransferase locus. A cause of postpartum coma. N Engl J Med. 1990;322(23):1652-1655.

8. Norman GR, Monteiro SD, Sherbino J, Ilgen JS, Schmidt HG, Mamede S. The Causes of Errors in Clinical Reasoning: Cognitive Biases, Knowledge Deficits, and Dual Process Thinking. Acad Med. 2017;92(1):23-30.

9. Graber ML, Franklin N, Gordon R. Diagnostic error in internal medicine. Arch Intern Med. 2005;165(13):14931499.

10. Smith SJ. EEG in neurological conditions other than epilepsy: when does it help, what does it add? J Neurol Neurosurg Psychiatry. 2005;76(Suppl 2):ii8-ii12.

11. Ebbinghaus H. Uber das Gedachtnis. Leipzig: Dunker. 1885.
12. Lee JJY, Wasserman WW, Hoffmann GF, van Karnebeek CDM, Blau N. Knowledge base and mini-expert platform for the diagnosis of inborn errors of metabolism. Genet Med. 2018;20(1):151-158.

13. Friedman CP, Gatti GG, Franz TM, Murphy GC, Wolf FM, Heckerling PS, Fine PL, et al. Do physicians know when their diagnoses are correct? Implications for decision support and error reduction. J Gen Intern Med. 2005;20(4):334-339.

14. Tarnopolsky M, Stevens L, MacDonald JR, Rodriguez C, Mahoney D, Rush J, Maguire J. Diagnostic utility of a modified forearm ischemic exercise test and technical issues relevant to exercise testing. Muscle Nerve. 2003;27(3):359366.

15. van't Leven M, Zielhuis GA, van der Meer JW, Verbeek AL, Bleijenberg G. Fatigue and chronic fatigue syndromelike complaints in the general population. Eur J Public Health. 2010;20(3):251-257.

16. Chinnery PF, Johnson MA, Wardell TM, Singh-Kler R, Hayes C, Brown DT, Taylor RW, et al. The epidemiology of pathogenic mitochondrial DNA mutations. Ann Neurol. 2000;48(2):188-193.

17. Murphy R, Turnbull DM, Walker M, Hattersley AT. Clinical features, diagnosis and management of maternally inherited diabetes and deafness (MIDD) associated with the $3243 \mathrm{~A}>\mathrm{G}$ mitochondrial point mutation. Diabet Med. 2008;25(4):383-399.

18. Smeets JGE, Brouwers MCGJ. Erfelijke stofwisselingsziekten bij volwassenen: wat men niet kent, herkent men niet? Bijblijven. 2016;32(8):558-565.

19. Adhikari AN, Gallagher RC, Wang Y, Currier RJ, Amatuni G, Bassaganyas L, Chen F, et al. The role of exome sequencing in newborn screening for inborn errors of metabolism. Nat Med. 2020;26(9):1392-1397.

20. Mamede S, van Gog $\mathrm{T}$, van den Berge K, Rikers RM, van Saase JL, van Guldener C, Schmidt HG. Effect of availability bias and reflective reasoning on diagnostic accuracy among internal medicine residents. JAMA. 2010;304(11):1198-1203.

21. Mamede S, Schmidt HG, Penaforte JC. Effects of reflective practice on the accuracy of medical diagnoses. Med Educ. 2008;42(5):468-475. 